\title{
PET/CT en cáncer pulmonar
}

\author{
DAVID LADRÓN DE GUEVARA H., RAÚL PEFAUR D.
}

\section{PET/CT in lung cancer}

Metabolic imaging studies are an integral part of oncology practice, particularly with 18 fluorodeoxyglucose PET scanning. Lung cancer is one of the primary indications of a PET/CT study. It is helpful in staging, evaluating treatment response and follow-up of these patients. The recent development of PET/CT, which incorporates a multislice CT scanner to the PET detector, improves results, combining metabolic information from the PET with anatomic data obtained with CT. It reduces false positive results from PET in cases of inflammatory disease such as pneumonia or drug reactions, which are frequent in this group of patients. These conditions are easily recognized by CT. It also improves the detection of primary tumors, when they are adjacent to atelectasis or desmoplastic reactions. PET-CT studies are able to characterize the metabolism of mediastinal and hilar lymph nodes, thus obviating the need for further related imaging studies or invasive procedures. In the assessment of metastatic disease, it allows a whole body analysis in only one study, with high predictive value and optimal cost-benefit relation. The detection of a second primary tumor is not infrequent in these patients. PET-CT is useful in the evaluation of treatment response after chemotherapy, and for the long term follow-up.

(Rev Med Chile 2010; 138: 1441-1450).

Key words: Lung neoplasms; Positron-emission tomography; Tomography scanners, $x$-Ray computed.

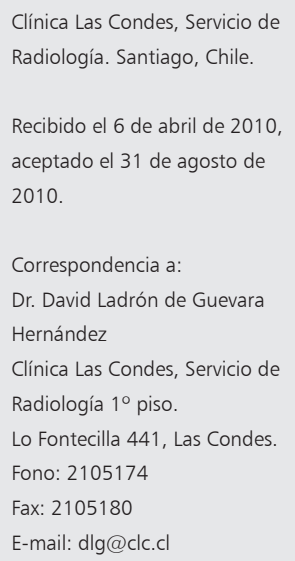

E 1 cáncer pulmonar es la principal causa de muerte por cáncer en Estados Unidos ${ }^{1}$. En Chile es la segunda causa de muerte por malignidad en hombres y quinta en mujeres ${ }^{2}$. El mayor factor de riesgo lo constituye el hábito de fumar tabaco, al cual está íntimamente relacionado. Otro factor de riesgo es la exposición a agentes cancerígenos, siendo el más conocido el asbesto ${ }^{3}$. Pacientes con EPOC y fibrosis pulmonar son también más susceptibles de desarrollar cáncer pulmonar ${ }^{4}$.

Desde el punto de vista patológico el cáncer pulmonar se clasifica en cáncer de células no pequeñas (CPCNP) (adenocarcinoma, cáncer de células escamosas, tumor de células grandes y otros subtipos) y cáncer de células pequeñas. Este último constituye un tipo de neoplasia más agresiva, de peor pronóstico y que requiere un enfoque terapéutico distinto. Por otro lado, etapificar correctamente un cáncer pulmonar es importante porque las opciones de tratamiento y pronóstico difieren significativamente en cada etapa ${ }^{5,4}$. En la actualidad, se utilizan varios métodos de imagen en el diagnóstico, evaluación y etapificación del cáncer pulmonar.

\section{Imágenes en cáncer pulmonar}

Habitualmente el primer examen que se realiza en la evaluación del cáncer pulmonar es una radiografía simple frontal y lateral del tórax. Este método es eficaz en el diagnóstico de lesiones avanzadas, pero está indudablemente limitado en la etapificación de la enfermedad como asimismo en la detección y clasificación de lesiones pequeñas.

La tomografía computada (TC) es un examen ampliamente extendido en la evaluación del tumor 
primario y en su extensión local y extra torácica. La óptima resolución espacial y buena resolución de contraste que se obtiene con tomógrafos multicorte hacen de esta técnica un método diagnóstico de gran rendimiento. Las características tomográficas de una lesión neoplásica primaria permiten en muchos casos aventurar con aceptable precisión el tipo histopatológico de un tumor ${ }^{7}$.

La resonancia magnética (RM) es otro examen que se utiliza en el estudio del cáncer pulmonar. Posee una mejor resolución de contraste que la tomografía computada, hecho que permite una adecuada evaluación de la relación del tumor con las estructuras mediastínicas y con la pared torácica, sin necesidad de administración de contraste endovenoso ${ }^{7}$. Sin embargo, su resolución espacial es significativamente inferior a la de la tomografía computada multicorte, la que a su vez permite la realización de reconstrucciones multiplanares isotrópicas de inmejorable calidad que hacen innecesarias las evaluaciones sagitales y coronales de la resonancia magnética. Otro factor a considerar es el mayor costo del examen de resonancia magnética en comparación con la tomografía computada.

Tanto la TC como la RM tienen la limitación que significa la identificación de compromiso metastásico de los ganglios linfáticos pulmonares, hiliares y mediastínicos. Es un hecho frecuente que linfonodos considerados normales por estos métodos diagnósticos (ganglios menores de 10 $\mathrm{mm}$ de diámetro en su eje menor) resultan comprometidos con tumor en el estudio histopatológico $^{6}$. También la presencia de derrame pleural, frecuente de observar en pacientes con cáncer pulmonar, sólo puede catalogarse como probablemente neoplásico si se asocia con engrosamiento o nodularidad de la pleura y/o si ésta se realza con el uso de contraste endovenoso ${ }^{7}$.

El advenimiento del PET (positron emission tomography) y especialmente del PET/CT (estudio híbrido de tomografía computada y de PET adquiridos simultáneamente) ha revolucionado el diagnóstico de la enfermedad neoplásica en general y del cáncer pulmonar en particular. La combinación de la información de la actividad metabólica de la lesión en estudio y su localización anatómica otorga a esta técnica una sensibilidad y especificidad que supera a todos los métodos diagnósticos hasta ahora utilizados en la evaluación del tumor pulmonar y su etapificación.

\section{Estudios metabólicos con PET}

Los estudios de imagen metabólicos forman parte integral de la oncología actual, y están representados principalmente por el PET. En oncología el radiotrazador más utilizado es la fluorodeoxiglucosa marcada con F18 (F18-FDG), que permite detectar tumores malignos, comúnmente hipermetabólicos. Este examen ha demostrado alta sensibilidad en la detección de dichas lesiones, aunque su especificidad suele ser menor debido a que existen varias otras condiciones que pueden mostrar captación de FDG, como por ejemplo algunos procesos inflamatorios ${ }^{8}$. Su dependencia de un examen morfológico complementario para una adecuada interpretación, asociado a su histórica escasa disponibilidad han obstaculizado su penetración en el uso clínico masivo. Los equipos diagnósticos híbridos (PET/CT, SPECT/CT) han constituido un significativo aporte en el ámbito médico, particularmente en el área oncológica, permitiendo obtener imágenes anatómicas y funcionales en un solo examen. Gracias a su optima relación costo/beneficio, y a su mayor disponibilidad actual, el PET/CT representa hoy por hoy una importante herramienta en el diagnóstico, etapificación y seguimiento de diversas neoplasias, particularmente del cáncer pulmonar, permitiendo obviar una serie de exámenes de etapificación, con el consiguiente ahorro de tiempo y recursos, y evitando cirugías innecesarias ${ }^{9-11}$.

\section{PET vs PET/CT}

La incorporación reciente del PET/CT - un detector PET acoplado a un tomógrafo computado multicorte- ha permitido mejorar notablemente el rendimiento de este estudio al combinar la información metabólica del PET con las imágenes anatómicas de la TC y acortar significativamente el tiempo de adquisición del examen, debido a la utilización del mapeo de atenuación de la TC para corregir la imagen del PET. La imagen fusionada de la TC ha permitido disminuir los falsos positivos del PET en patología inflamatoria pulmonar muy frecuente en estos pacientes (neumopatias agudas, neumopatia obstructiva, reacción a drogas), las que son fácilmente diferenciables de una masa utilizando TC (Figura 1), y caracterizar mejor el tumor primario, a veces difícil de separar del com- 




Figura 1. Paciente portador de un cáncer pulmonar (no visualizado en estos cortes) con múltiples áreas de relleno alveolar que concentran FDG, compatibles con focos bronconeumónicos. Enfisema paraseptal y centroacinar.

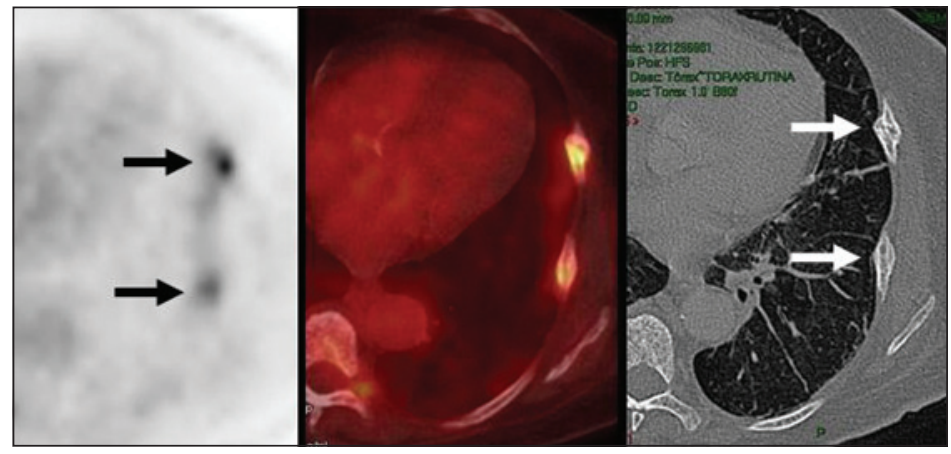

Figura 3. Captación de origen benigno en parrilla costal izquierda que corresponden a fracturas en ángulos costales visibles en tomografía.

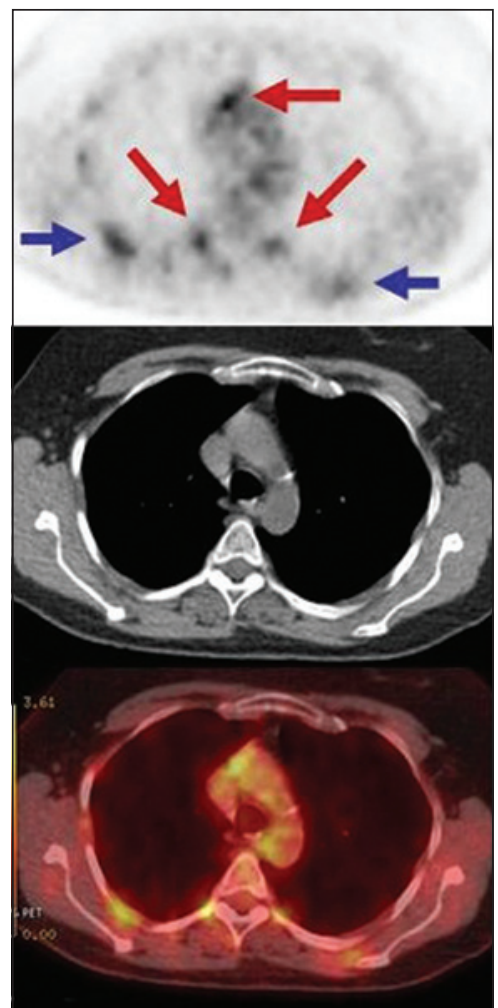

Figura 2. Focos de grasa parda ("brown fat") hipermetabólica no patológica a nivel mediastínico y paravertebral (Flechas rojas). Captación fisiológica en músculos intercostales y dorsales (Flechas azules). La imagen morfológica del CT y la fusión con el PET permite adecuada correlación anatómica. promiso atelecatásico o desmoplástico adyacente. El PET/CT hace posible también reconocer correctamente la "grasa parda" hipermetabólica, no patológica, de frecuente visualización en el tórax ${ }^{12}$ (Figura 2). La fusión con la TC es particularmente útil en reconocer lesiones benignas en hueso que pueden mostrar grados de captación de F18-FDG, como fracturas, cambios inflamatorios o degenerativos (Figura 3), aumentando la especificidad del método.

\section{Cuantificación de la captación de FDG}

El SUV (Standarized uptake value) es el índice más utilizado para cuantificar la captación de FDG en una lesión, y refleja su grado de metabolismo. Esto es de suma importancia ya que se ha establecido que la intensidad de captación tiene relación con la desdiferenciación celular y por lo tanto, agresividad del tumor, lo que le confiere a este índice un valor pronóstico intrínseco ${ }^{13}$. 
Además su uso eleva el rendimiento del método, principalmente aumentando la especificidad ya que constituye una herramienta útil en diferenciar lesiones benignas de malignas según su captación. Ha demostrado una alta reproducibilidad inter e intraobservador al ser calculada, y se define con la siguiente fórmula ${ }^{14}$ :

SUV: $\frac{\text { Actividad en una lesión (Bq) / Volumen píxel (ml) }}{\text { Actividad inyectada (Bq) / Peso pcte (gr) }}$

El SUV tiene gran utilidad pronóstica antes y después del tratamiento, y permite comparar estudios entre sí, objetivando los efectos metabólicos de la terapia. Su medición considerando la superficie corporal o la masa muscular ha demostrado ser más consistente que la que incluye sólo el peso en esta fórmula.

\section{Etapificación T}

El PET/CT presta gran utilidad en el estudio de la invasión tumoral de pleura, mediastino y vía aérea mayor ${ }^{15}$. La existencia de derrame o engrosamiento pleural requiere siempre un estudio adicional para descartar compromiso neoplásico, muchas veces de tipo invasivo, con toma de citología o biopsia. La captación de FDG pleural es siempre patológica y en un contexto neoplásico pulmonar es altamente sugerente de un origen secundario (Figura 4). En tumores con compromiso de mediastino la utilización de contraste iodado endovenoso permite estudiar de mejor manera la relación del tumor con los grandes vasos y el pericardio, y su resecabilidad. La capacidad de diferenciar tejido tumoral metabólicamente activo de parenquima atelectasico es vital en la planeación de la radioterapia, modificando el campo de radiación en hasta 30 a $40 \%$ de los pacientes ${ }^{16}$. En tumores asociados a neumonías postobstructivas y masas con extensas áreas de necrosis el PET/CT aporta valiosa información para ubicar el sitio de toma de biopsia (Figura 5).

\section{Etapificación N}

El PET/CT hace posible caracterizar metabólicamente los linfonodos hiliares y mediastínicos mejor que cualquier otra técnica de imagen, ya que logra identificar ganglios pequeños hipermetabólicos, negativos bajo los criterios morfológicos de la TC, así como también linfonodos aumentados de tamaño pero sin o con baja captación, frecuentemente reactivos o residuales sin compromiso neoplásico ${ }^{6}$ (Figura 6). Un tumor pulmonar ávido de FDG con mediastino metabólicamente negativo tiene un valor predictivo negativo de $99 \%{ }^{17}$. El PET/CT reduce los costos y el tiempo de estudio en etapificación ganglionar, evitando procedimeintos adicionales de estudio (RM, ultrasonografia o punción transesofágica o transbronquial) y en muchos casos la mediastinoscopia ${ }^{17-19}$. Por otro lado, el mapeo metabólico ganglionar hace más fácil y directa la evaluación cito o histológica en caso de requerir estudio confirmatorio.

\section{Etapificación M}

En CPCNP el PET/CT permite detectar metástasis extratoráxicas en 10-20\% de los pacientes con estudio convencional negativo, con un obvio impacto en el tratamiento ${ }^{20}$. Es más sensible en detección de metástasis óseas líticas que el cintigrama óseo, de mayor rendimiento que la TC en evaluación de nódulos suprarrenales indeterminados (Figura 7), y más sensible en lesiones secundarias hepáticas (Figura 8) 21,22. La imagen fusionada morfofuncional aumenta significativamente la especificidad del método, permitiendo identificar la captación fisiológica en intestino y vías urinarias. Cabe destacar su bajo rendimiento en lesiones secundarias cerebrales dado la alta captación de FDG del parénquima cerebral normal, lo que enmascara las lesiones metastásicas. Por este motivo se recomienda complementar el estudio con RM ó TC con contraste cerebral en todo paciente con una neoplasia pulmonar ${ }^{5,6,23}$.

\section{Detección de un segundo tumor primario}

Seis a diecinueve porciento de los pacientes con cáncer pulmonar tienen un segundo cáncer extrapulmonar al momento del diagnóstico ${ }^{24-26}$. Setenta porciento de estas neoplasias se asocian a tabaco y corresponden principalmente a cáncer de cabeza y cuello, tumores del tracto digestivo (Figura 9), y neoplasias de vías urinarias ${ }^{25,27}$. Sin embargo, ante un paciente con cáncer de pulmón y un foco hipercaptante extrapulmonar único, 54\% de los casos se tratará de una metástasis y sólo 19\% de una segunda neoplasia ${ }^{26}$, por lo que se requerirá en muchos casos confirmación histológica de la lesión. 


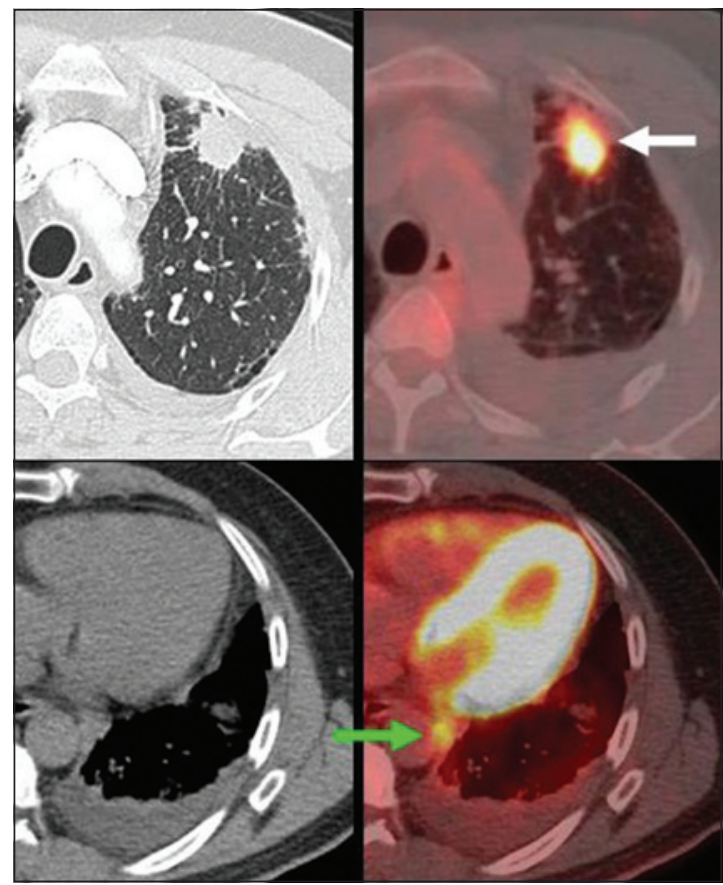

Figura 4. Nódulo pulmonar espiculado hipercaptante en lóbulo superior izquierdo (flecha blanca), con biopsia por punción que muestra adenocarcinoma moderadamente diferenciado. Nódulo pleural hipermetabólico paraaórtico izquierdo asociado a derrame y leve captación de FDG pleural (flecha verde), con citología de liquido pleural positiva para células neoplásicas.

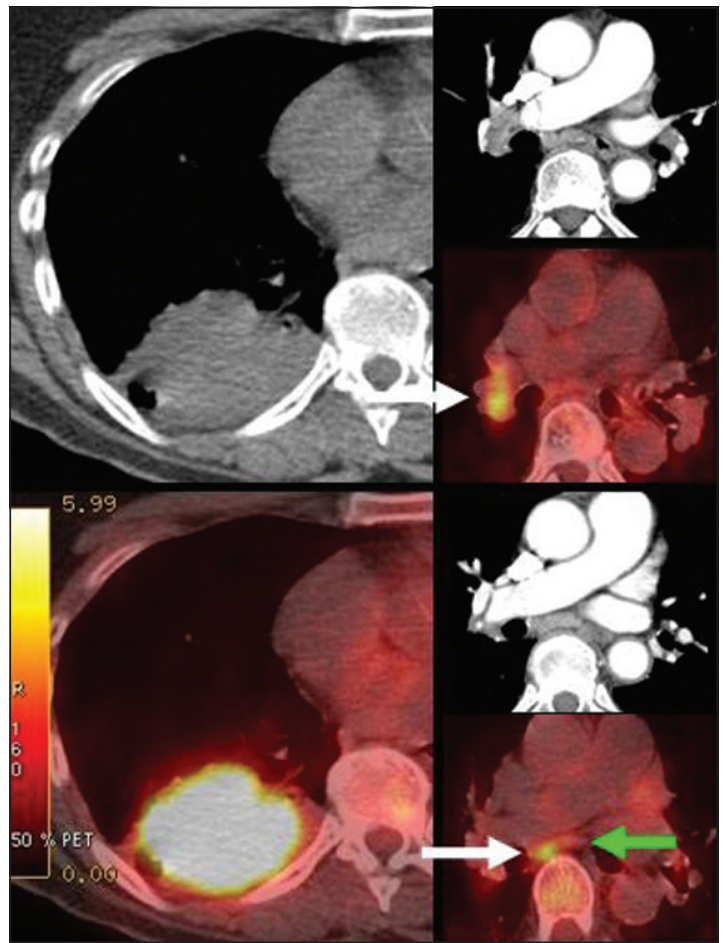

Figura 6. Carcinoma escamoso en lóbulo inferior derecho con dos pequeñas adenopatías hipercaptantes en hilio y mediastino (flecha blanca). Se resecaron 23 ganglios con 2 de ellos positivos (hiliar y subcarinal). Pequeño ganglio subcarinal izquierdo hipocaptante, sin compromiso neoplásico (flecha verde).

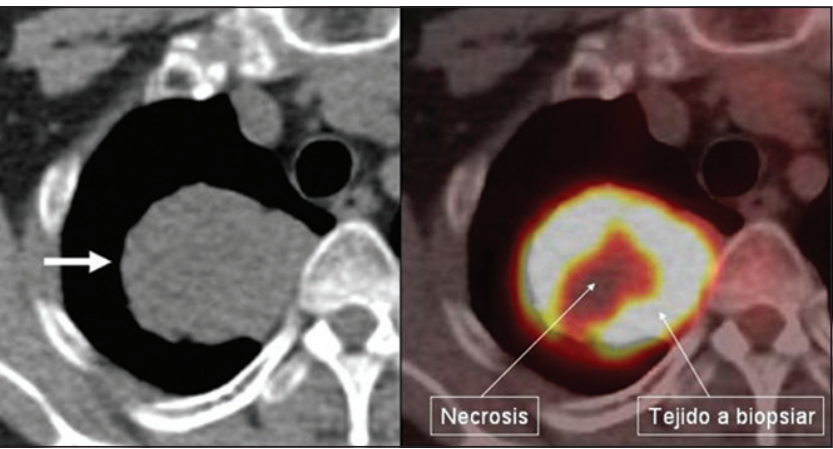

Figura 5. Cáncer pulmonar de células grandes del lóbulo superior derecho con extensa necrosis central y periferia ávida de FDG (SUVmax: 24,0). La biopsia debe ser tomada de la periferia de la lesión para obtener una muestra adecuada y evitar las áreas necróticas.

\section{Cáncer pulmonar de células pequeñas $(\mathrm{CPCP})$}

$\mathrm{Al}$ igual que en el CPCNP, el PET/CT constituye el método de elección para la etapificación, y es superior al estudio de estadiaje convencional en todas las localizaciones excepto el cerebro, siendo particularmente útil en compromiso ganglionar mediastínico ${ }^{28}$ (Figura 10). El PET/CT detecta correctamente compromiso ganglionar regional no visible por los métodos convencionales en 25\% de los pacientes, con significativa modificación del campo de radioterapia en todos ellos $^{29}$. Constituye una herramienta de gran valor en la planeación de la radioterapia, definiendo de manera precisa el "target" a irradiar. 


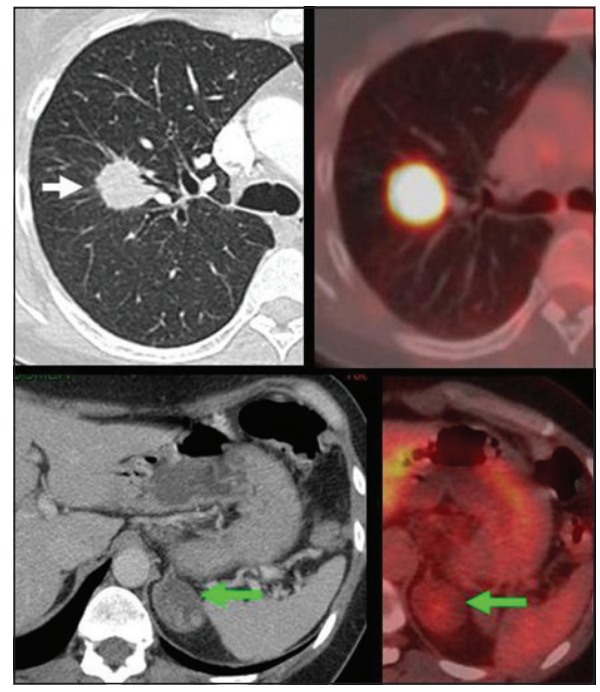

Figura 7. Masa en lóbulo superior derecho (flecha blanca) que muestra intensa captación de FDG, con SUVmax de 17,5. El paciente también presenta un nódulo suprarrenal izquierdo (flecha verde) que en el PET mostró escasa captación (SUVmax: 2,4), por lo tanto de comportamiento benigno. Ambas lesiones se resecaron en el mismo acto operatorio, con resultado maligno en pulmón (Ca escamoso) y benigno en glándula suprarrenal (adenoma suprarrenal).

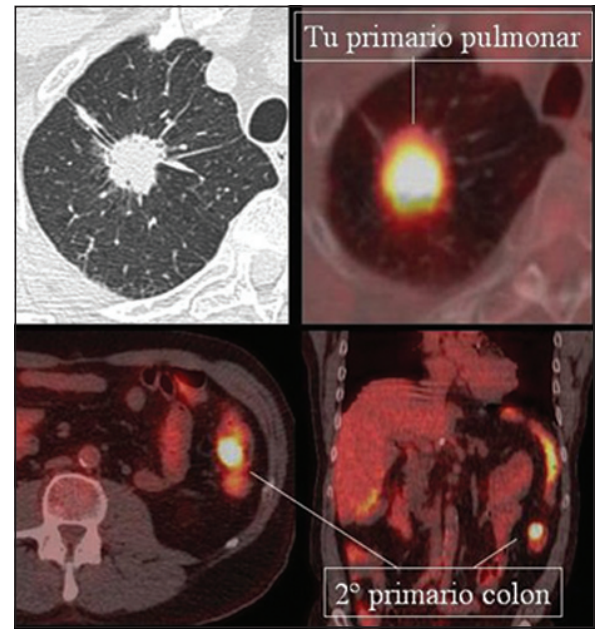

Figura 9. Masa espiculada apical derecha hipermetabólica. Nódulo hipercaptante en colon izquierdo. Primera cirugía: lobectomía superior derecha con biopsia positiva para adenocarcinoma pulmonar tipo micropapilar. Segunda cirugía 1,3 meses después: hemicolectomia izquierda con biopsia positiva para adenocarcinoma de colon tipo tubular moderadamente diferenciado.

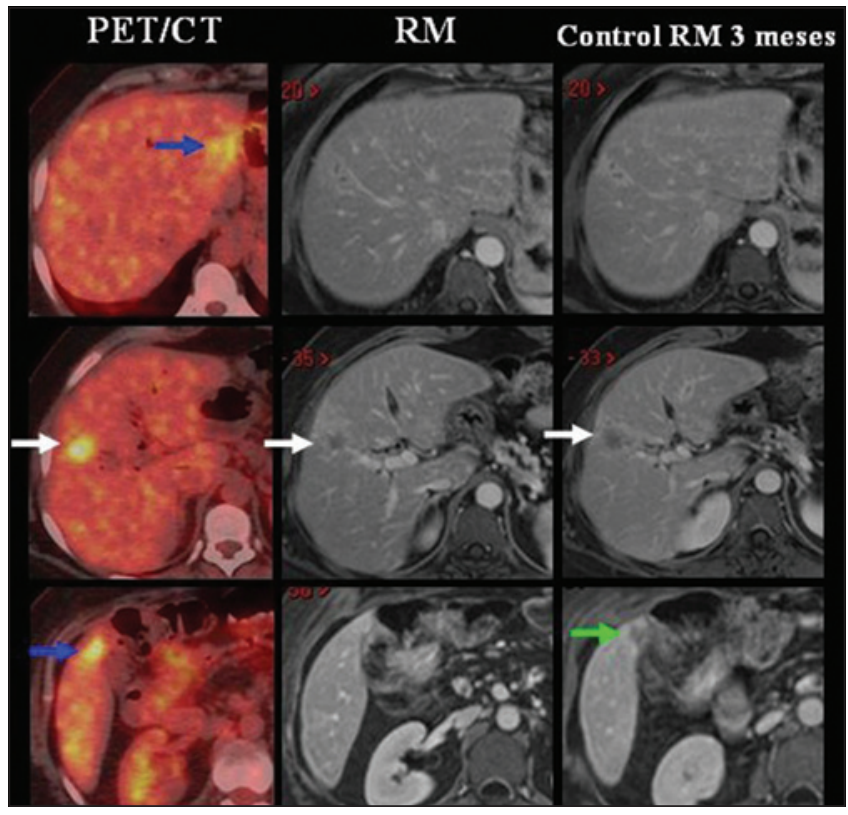

Figura 8. Metástasis hepáticas visibles en PET/CT, algunas de ellas no evidentes en RM contemporánea (flecha azul). En control con RM a los 3 meses se hace visible la lesión del segmento V-VI (flecha verde), y se observa crecimiento de otras lesiones (flecha blanca).

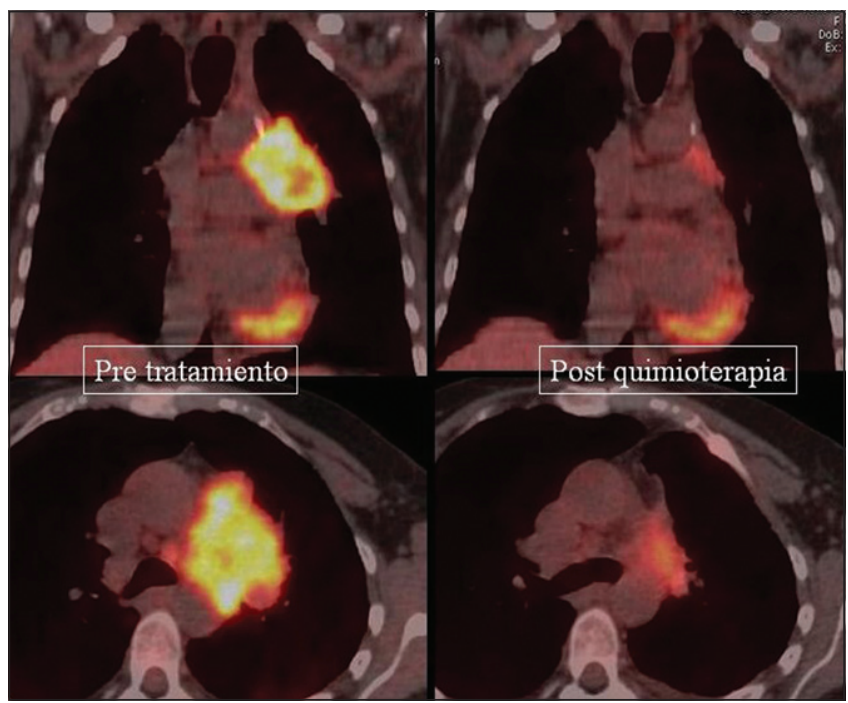

Figura 10. Carcinoma pulmonar de células pequeñas suprahiliar izquierdo. Control tardío post quimioterapia (finalizado el tratamiento) muestra respuesta metabólica casi completa, lo que determina un relativo mejor pronostico. 
PET/CT en cáncer pulmonar - D. Ladrón de Guevara H. et al

\section{Control de tratamiento y pronóstico}

El índice SUV de captación pre tratamiento en el tumor maligno se correlaciona inversamente con la sobrevida tanto en $\mathrm{CPCNP}^{13}$ como en $\mathrm{CPCP}^{30}$. La respuesta metabólica precoz (interciclos) y tardía (terminado el tratamiento) a la quimioterapia tiene implicancias pronósticas bien demostradas en cáncer pulmonar (Figura 10). Ya desde el primer ciclo de quimioterapia se pueden dibujar curvas de sobrevida de acuerdo al grado de disminución de metabolismo glucídico en el tumor o en las adenopatías, cuantificado mediante SUVmax ${ }^{31}$. Para un adecuado control tardío postquimioterapia el PET/CT debe ser realizado al menos 3-4 semanas después de terminado el tratamiento. En el caso de la radioterapia el control debe realizarse al menos 4 a 6 meses posterior al tratamiento para evitar falsos positivos determinados por los extensos cambios inflamatorios propios de este tratamiento.

\section{Nódulo pulmonar solitario}

Los nódulos pulmonares únicos o múltiples son hallazgos de gran frecuencia tanto en pacientes fumadores como no fumadores ${ }^{32,33}$, y su detección se ha optimizado notablemente con la aparición de equipos de tomografía computada multidetectores, que permiten una adquisición volumétrica rápida con cortes de grosores cercanos a $1 \mathrm{~mm}$. La significación de dichos nódulos tiene estrecha relación con factores clínicos (edad del paciente, hábito tabáquico, enfermedad maligna conocida), y con las características propias del nódulo (tamaño, bordes espiculados, ubicación en lóbulos superiores, etc) ${ }^{34}$. Existen múltiples modelos que se han propuesto en el estudio y seguimiento de los nódulos pulmonares, incluyendo la mayoría de ellos la TC y el PET, además de procedimientos de tipo invasivo, con seguimiento imagenológico por al menos 2 años $^{34-36}$. Algunos autores han destacado la utilidad de otros estudios de menor uso clínico en el estudio de nódulos pulmonares, como la RM y SPECT Tc99m-depreotide, describiendo rendimientos similares al estudio metabólico ${ }^{37}$. Actualmente, el PET/CT se ha incluido en el estudio de los nódulos sólidos de $7 \mathrm{~mm}$ o más, ya que se ha demostrado su costo/eficacia en determinar si un nódulo requiere estudio histológico o no, especialmente en aquellos con probabilidad pre- test intermedia para malignidad ${ }^{38}$. Su capacidad de evaluar el metabolismo glucídico o consumo de glucosa mediante la cuantificación del grado de captación de FDG de estas lesiones permite discriminar entre nódulos benignos (sin o baja captación) y malignos (alta captación) con una razonable eficiencia ${ }^{39}$. Excepciones a esto suceden con nódulos benignos de etiología inflamatoria granulomatosa que pueden mostrar captación significativa de FDG y por otro lado, con nódulos de naturaleza neoplásica como los carcinoides típicos $\mathrm{y}$ algunos adenocarcinomas bien diferenciados que característicamente presentan bajo metabolismo glucídico, con índices SUV incluso menores de 2,5. Sin embargo, un nódulo que no presente captación alguna tiene un valor predictivo negativo mayor al $97 \%$ y debe considerarse benigno ${ }^{40,41}$. Indices de SUVmax $>2,5$ tiene un valor predictivo positivo de malignidad mayor de $50 \%$.

\section{Costo/beneficio del PET/CT}

El estudio del impacto económico del PET en cáncer pulmonar ha sido abordado por algunos autores, y aunque se ha demostrado su amplia superioridad sobre las otras técnicas diagnósticas de imagen y su menor costo relativo al disminuir otros procedimientos, su relación costo/beneficio no ha sido plenamente establecida ${ }^{11}$. Diversas publicaciones demuestran el alto impacto del PET en el manejo de los pacientes con cáncer pulmonar versus el estudio convencional, produciendo cambios mayores en el tratamiento en hasta $63 \%$ de los $\operatorname{casos}^{42}$. En un estudio multicéntrico encabezado por Hillner se demuestra que en la mayor parte de estos casos el PET incentiva el tratamiento paliativo y curativo en pacientes que en ausencia de PET sólo recibirían medidas de soporte ${ }^{10}$. Por otro lado, el uso del PET/CT disminuye significativamente el número total de toracotomías y de toracotomías innecesarias en pacientes con cáncer pulmonar 9 . Posee un mayor rendimiento global que todas las otras técnicas diagnósticas juntas, especialmente en etapificación $\mathrm{N}$ y $\mathrm{M}$. Esto es particularmente evidente en el estudio de mediastino, pudiéndose obviar la mediastinoscopia cuando el PET muestra mediastino negativo y un tumor primario pulmonar ávido de $\mathrm{FDG}^{17,43}$, o enfocando el estudio invasivo directamente hacia las lesiones metabólicamente activas. Esto lógicamente significa una 
agilización y menor costo del estudio de etapificación al disminuir drásticamente el número de tests imagenológicos y procedimientos invasivos que se le practican al paciente. De esta manera se puede iniciar precozmente el tratamiento al evitar procedimientos y cirugías innecesarias.

Una de las limitaciones de la técnica PET/CT sigue siendo su menor disponibilidad respecto a otros métodos diagnósticos, y su dependencia directa con un ciclotrón, donde se produce el radiofármaco F18-FDG. Sin embargo, la implementación explosiva de esta tecnología en el mundo ha acortado distancias rápidamente con otras técnicas ${ }^{44}$, y posiblemente en el futuro cercano el estudio metabólico con PET/CT llegue a ser tan común como la RM y el SPECT son en la actualidad.

\section{Conclusión}

El PET/CT con F18-FDG es de gran utilidad en el diagnóstico, etapificación, control de tratamiento y seguimiento en cáncer pulmonar. En centros con esta técnica disponible se recomienda su utilización rutinaria en esta patología y en el estudio de nódulos pulmonares.

\section{Referencias}

1. Howe HL, Wingo PA, Thun MJ, Ries LAG, Rosenberg HM, Feigal EG, et al. Annual Report to the Nation on the Status of Cancer (1973 Through 1998), Featuring Cancers With Recent Increasing Trends. J Natl Cancer Inst 2001; 93: 824-42.

2. Programa Nacional de Cáncer. MINSAL 2001. Reactualizado el 2004. http://www.redsalud.gov.cl/archivos/ cancer/prcancernino.pdf.

3. Ullrich RL. Etiology of cancer: Physical factors. In: DeVita VT Jr., Hellman S, Rosenberg SA, editors. Cancer: Principles and Practice of Oncology. Vol. 1 and 2. 7th ed. Philadelphia, USA: Lippincott Williams and Wilkins, 2004: 597-618.

4. Webb WR. Lung and Bronchopulmonary Neoplasms. En: Webb WR, Higgins CB. Thoracic Imaging. Pulmonary and Cardiovascular Radiology. Philadelphia, USA. Lippincott, Williams and Wilkins, 2005: 66-111.

5. Rivera MP, Mehta AC. Initial Diagnosis of Lung Cancer. ACCP Evidence-Based Clinical Practice Guidelines (2nd Edition). CHEST 2007; 132: 131S-148S.
6. Silvestri GA, Gould MK, Margolis ML, Tanoue LT, McCrory D, Toloza E, et al. Noninvasive Staging of Nonsmall Cell Lung Cancer. ACCP Evidenced-Based Clinical Practice Guidelines (2nd Edition). CHEST 2007; 132: 178S-201S.

7. Lung Cancer. In: Naidich DP, Webb WR, Müller NL, Vlahos I, Krinsky GA. Computed Tomography and Magnetic Resonance of the Thorax. Philadelphia, USA. Wolters Kluwer/Lippincott, Williams and Wilkins, Fourth Edition, 2007: 621-69.

8. Asad S, Aquino SL, Piyavisetpat N, Fischman AJ. FalsePositive FDG Positron Emission Tomography Uptake in Nonmalignant Chest Abnormalities. AJR 2004; 182: 983-9.

9. Fischer B, Lassen U, Mortensen J, Larsen S, Loft A, Bertelsen A, et al. Preoperative Staging of Lung Cancer with Combined PET-CT. N Engl J Med 2009; 361: 32-9.

10. Hillner B, Siegel BA, Liu D, Shields AF, Gareen IF, Hanna L, et al. Impact of Positron Emission Tomography/Computed Tomography and Positron Emission Tomography (PET) Alone on Expected Management of Patients With Cancer: Initial Results From the National Oncologic PET Registry. J Clin Oncol 2008; 26: 2155-61.

11. Buck AK, Herrmann K, Stargardt T, Dechow T, Krause BJ, Schreyogg J. Economic Evaluation of PET and PET/ CT in Oncology: Evidence and Methodologic Approaches. J Nucl Med 2010; 51: 401-12.

12. Truongl MT, Erasmus JJ, Munden RF, Marom EM, Sabloff BS, Gladish GW, et al. Focal FDG Uptake in Mediastinal Brown Fat Mimicking Malignancy: A Potential Pitfall Resolved on PET/CT. AJR 2004;183:1127-32.

13. Downey RJ, Akhurst T, Gonen M, Vincent A, Bains MS, Larson S. Preoperative F-18 Fluorodeoxyglucose-Positron Emission Tomography Maximal Standardized Uptake Value Predicts Survival After Lung Cancer Resection. J Clin Oncol 2004; 22: 3255-60.

14. Lin EC, Alavi A, Kinahan P. Standarized uptake value. En: Lin EC, Alavi A. PET and PET/CT: A clinical guide. New York, USA: Thieme Medical Publishers Inc; 2005: 28-31.

15. Lardinois D, Weder W, Hany TF, Kamel EM, Korom S, Seifert B, et al. Staging of non-small-cell lung cancer with integrated positron-emission tomography and computed tomography. N Engl J Med 2003; 348: 2500-7.

16. Dizendorf EV, Baumert BG, von Schulthess GK, Lu"tolf UM, Steinert HC. Impact of whole-body 18F-FDG PET on staging and managing patients for radiation therapy. J Nucl Med 2003; 44: 24-9.

17. Enoch Lee B, Redwine J, Foster C, Abella E, Lown T, Lau $\mathrm{D}$, et al. Mediastinoscopy might not be necessary in patients with non-small cell lung cancer with mediastinal 
lymph nodes having a maximum standardized uptake value of less than 5,3. J Thorac Cardiovasc Surg 2008; 135: 615-9.

18. Schimmer C, Neukam K, Elert O. Staging of non-small cell lung cancer: clinical value of positron emission tomography and mediastinoscopy. Interact CardioVasc Thorac Surg 2006; 5: 418-23.

19. Bryant AS, Cerfolio RJ, Klemm KM, Ojha B. Maximum Standard Uptake Value of Mediastinal Lymph Nodes on Integrated FDG PET-CT Predicts Pathology in Patients with Non-Small Cell Lung Cancer. Ann Thorac Surg 2006; 82: 417-23.

20. Goerres GW, von Schulthess GK, Steinert HC. Why Most PET of Lung and Head-and-Neck Cancer Will Be PET/ CT. J Nucl Med 2004; 45: 66S-71S.

21. Hellwig D, Ukena D, Paulsen F, Bamberg M, Kirsch CM. A meta-analysis of the utility of FDG-PET in lung tumors. [German]. Pneumologie 2001; 55: 367-77.

22. Marom EM, McAdams HP, Erasmus JJ. Staging nonsmall cell lung cancer with whole-body PET. Radiology 1999; 212: 803-9.

23. Samson DJ, Seidenfeld J, Simon GR, Turrisi AT, Bonnell C, Ziegler KM, et al. Evidence for Management of Small Cell Lung Cancer. ACCP Evidence-Based Clinical Practice Guidelines (2nd Edition). CHEST 2007; 132:314S$323 S$.

24. Westreenen HL, Westerterp M, Jager PL, van Dullemen HM, Sloof GW, Comans E, et al. Synchronous Primary Neoplasms Detected on 18F-FDG PET in Staging of Patients with Esophageal Cancer. J Nucl Med 2005; 46: 1321-5.

25. Duchateau CSJ, Stokkel MPM. Second Primary Tumors Involving Non-small Cell Lung Cancer. Chest 2005; 127: 1152-8.

26. Lardinois D, Weder W, Roudas M, von Schulthess GK, Tutic M, Moch H, et al. Etiology of Solitary Extrapulmonary Positron Emission Tomography and Computed Tomography Findings in Patients With Lung Cancer. J Clin Oncol 2005; 23: 6846-53.

27. Ladrón de Guevara D, Pefaur R, Pardo C. Neoplasias insospechadas detectadas en pacientes que se realizaron PET/CT de cuerpo entero. Rev Med Clin Condes 2010; 21 (En prensa).

28. Kamel EM, Zwahlen D, Wyss MT, Stumpe KDM, von Schulthess GK, Steinert HC. Whole-body 18F-FDG PET improves the management of patients with small-cell lung cancer. J Nucl Med 2003; 44: 1911-7.

29. Bradley JD, Dehdashti F, Mintun MA, Govindan R, Trinkaus K, Siegel BA. Positron Emission Tomography in Limited-Stage Small-Cell Lung Cancer: A Prospective Study. J Clin Oncol 2004; 22: 3248-54.
30. Lee YJ, Cho A, Cho BC, Yun M, Kim SK, Chang J, et al. High Tumor Metabolic Activity as Measured by Fluorodeoxyglucose Positron Emission Tomography Is Associated with Poor Prognosis in Limited and Extensive Stage Small-Cell Lung Cancer. Clin Cancer Res 2009; 15: 2426-32.

31. Hoekstra CJ, Stroobants SG, Smit EF, Vansteenkiste J, van Tinteren H, Postmus PE, et al. Prognostic Relevance of Response Evaluation Using [18F]-2-Fluoro-2-DeoxyD Glucose Positron Emission Tomography in Patients With Locally Advanced Non Small-Cell Lung Cancer. J Clin Oncol 2005; 23: 8362-70.

32. Lillington GA. Management of solitary pulmonary nodules. Dis Mon 1991; 37: 271-318.

33. New York Early Lung Cancer Action Project Investigators. CT Screening for Lung Cancer: Diagnoses Resulting from the New York Early Lung Cancer Action Project. Radiology 2007; 243: 239-49.

34. Soubani AO. The evaluation and management of the solitary pulmonary nodule. Postgrad Med J 2008; 84: 459-66.

35. Gould MK, Fletcher J, Iannettoni MD, Lynch WR, Midthun DE, Naidich DP, et al. Evaluation of Patients With Pulmonary Nodules: When Is It Lung Cancer? ACCP Evidence-Based Clinical Practice Guidelines (2nd Edition). CHEST 2007; 132: 108S-30S.

36. MacMahon H, Austin JHM, Gamsu G, Herold CJ, Jett JR, Naidich DP, et al. Guidelines for Management of Small Pulmonary Nodules Detected on CT Scans: A Statement from the Fleischner Society. Radiology 2005; 237: 395-400.

37. Cronin P, Dwamena BA, Kelly AM, Carlos RC. Solitary Pulmonary Nodules: Meta-analytic Comparison of Cross-sectional Imaging Modalities for Diagnosis of Malignancy. Radiology 2008; 246: 772-82.

38. Gould MK, Sanders GD, Barnett PG, Rydzak CE, Maclean CC, McClellan MB et al. Cost-effectiveness of alternative management strategies for patients with solitary pulmonary nodules. Ann Intern Med 2003; 138: 724-35.

39. Kim SK, Allen-Auerbach M, Goldin J, Fueger BJ, Dahlbom M, Brown M, et al. Accuracy of PET/CT in Characterization of Solitary Pulmonary Lesions. J Nucl Med 2007; 48: 214-20.

40. Fletcher JW, Kymes SM., Gould M, Alazraki N, R. Coleman RE, Lowe VJ, et al. A Comparison of the Diagnostic Accuracy of 18F-FDG PET and CT in the Characterization of Solitary Pulmonary Nodules. J Nucl Med 2008; 49: 179-85.

41. Hashimoto Y, Tsujikawa T, Kondo C, Maki M, Momose M, Nagai A, et al. Accuracy of PET for Diagnosis of Solid 
Pulmonary Lesions with 18F-FDG Uptake Below the Standardized Uptake Value of 2.5. J Nucl Med 2006; 47: 426-31.

42. Bunyaviroch T, Coleman RE. PET Evaluation of Lung Cancer. J Nucl Med 2006; 47: 451-69.

43. Vansteenkiste JF. FDG-PET for lymph node staging in
NSCLC: a major step forward, but beware of the pitfalls. Lung Cancer 2005; 47: 151-3.

44. Basu S, Alavi A. Revolutionary impact of PET and PET$\mathrm{CT}$ on the day-to-day practice of medicine and its great potential for improving future health care. [Editorial, Review] Nucl Med Rev Cent East Eur 2009; 12: 1-13. 